\title{
ARTICLE
}

\section{Shielding calculation for the CSNS target station}

\author{
Wen Yin ${ }^{\mathrm{a}}$, Tai-ran Liang ${ }^{\mathrm{a}, \mathrm{b}}$, Fei Shen ${ }^{\mathrm{a}, \mathrm{c}}$, Songlin Wang ${ }^{\mathrm{d}}$, Quan-zhi Yu ${ }^{\mathrm{a}}$ and Tian-jiao Liang ${ }^{\mathrm{a} *}$ \\ ${ }^{a}$ Engineering Centre of CSNS Target Station and Instruments, Institute of Physics, Chinese Academy of Sciences, Beijing 100190, \\ China; ${ }^{b}$ Nankai University, Tianjing300071, China; ${ }^{c}$ Lanzhou University, Lanzhou 730000, China; ${ }^{d}$ Division for Experimental \\ Physics, Institute of High Energy Physics, Chinese Academy of Sciences, Beijing 100049,China
}

In this paper we firstly calculate the one-dimension shielding models to give the basic data for the Chinese Spallation Neutron Source (CSNS) target station. Then the three-dimensional shielding calculations have been performed for the detailed design of the CSNS target station. The gaps and void spaces in the CSNS target station are considered for precise estimation. We find that 4.8-meter-radius steel adding 1.2-meter-thick magnetite concrete can satisfy the shielding requirement in the horizontal direction. The dose rate on the top of the ceiling is a little higher than $2.5 \mu \mathrm{Sv} / \mathrm{h}$ due to streaming effect, which originates from the moderator transfer lines. A thin sandwich structure will be adopted for decreasing residual radioactivity dose rate on the altitude directions after considering the engineering cost and maintenance. All these calculations lie on the Monte Carlo simulation code MCNPX2.5.0.

Keywords: CSNS; target station; shielding; Monte Carlo; dose rate

\section{Introduction}

The Chinese Spallation Neutron Source (CSNS) is a $100 \mathrm{~kW}$-level spallation neutron source on its phase one and can be upgraded to $500 \mathrm{~kW}$-level on its phase two [1-3]. It had entered the stage of construction in September, last year.

When the proton beams bombard the tungsten target, the high-energy neutrons are extremely penetrating. A well-designed shielding is needed to prevent them from causing excessive biological dose rates [2,3]. It is important that structural shielding be properly designed and installed in the original construction because correction or additions, after facilities are completed, are usually expensive. We had used the deterministic discrete ordinates transport code DOORS[4], HILO2K[5] and the newly developed cross-section library HEST1.0[6] to perform the two-dimensional CSNS target station shielding design, which gave us some conceptual and important data[7]. But many gaps and void spaces are involved in the target station such as gaps between bulk shielding and shutters. In this case, the Monte Carlo particle transport code MCNPX with a variety of variance reduction technique is more suited for shielding design.

In this paper, we calculate the one-dimension models to give a basic data for the CSNS target station. Then we perform the detailed three-dimension shielding design for the CSNS target station. All these calculation are finished by the code MCNPX2.5.0 [8]. The Bertini INC physics model is used for simulation of high-energy particle transport and the LA150n and LA150h libraries are used. When tabular nuclear data are available, use them up to their upper limit for each nuclide. The dimensions are reviewed many times according to calculated results. The weight window generator and geometrical splitting are used to reduce the computer time.

\section{One-dimensional spherical calculation results}

The target radiation dose rates of $2.5 \mu \mathrm{Sv} / \mathrm{h}$ and $25 \mu$ $\mathrm{Sv} / \mathrm{h}$ are used in the CSNS project user area and target station high-bay area respectively. All calculations are for $500 \mathrm{~kW}$ beam power. We give the distributions of neutrons around the bare tungsten target when the target is bombarded by $1.6 \mathrm{GeV}$ protons (see Figure 1). From Figure 1 we can see that the energies of the neutrons depend highly on the escaping direction. The neutrons around the target are relatively hard especially in the forward direction which will approach the energy of the incident proton. In this paper we choose the neutrons in the forward direction as the source term.

Using simple one-dimensional transport calculation is very convenient in the preliminary stages of the CSNS target station design. Firstly one dimensional spherical model is used to give a conceptual design. Neutrons

\footnotetext{
*Corresponding author. Email: tjliang@iphy.ac.cn
} 




Figure 1 . The high energy neutron spectra around the target in the different directions (here $30 \sim 45^{\circ}$ means the degree to the proton beam incident direction).

escape from the tungsten target surface when it is bombarded by $1.6 \mathrm{GeV}$ proton beam. These neutrons whose directions are in the range from 30 45 degree to the proton beam are set as the surface source term. After considering the moderator, reflector and the void space, the sphere surface is determined to be distant from the center 0.5 meter. As a shield against high-energy neutrons, steel can be quite effective if it is followed by a material made of light elements, such as concrete. Magnetite concrete is more effective for photon shielding than concrete not only because of the increased density, but also because of the $\mathrm{Z}$ dependence of the photoelectric and pair-production cross sections. Thus when we consider the neutron and photon shielding we choose three materials steel, magnetite concrete and concrete. Weight densities of $7.4 \mathrm{~g} / \mathrm{cm}^{3}, 3.60 \mathrm{~g} / \mathrm{cm}^{3}$, and $2.35 \mathrm{~g} / \mathrm{cm}^{3}$ are used for steel $(0.2 \% \mathrm{C}, 0.1 \% \mathrm{Cr}, 0.2 \% \mathrm{Ni}$, $0.1 \% \mathrm{Cu}, 0.25 \% \mathrm{Si}, 0.45 \% \mathrm{Mn}, 98.7 \% \mathrm{Fe})$, magnetite concrete $(47.4 \% \quad \mathrm{Fe}, \quad 33.1 \% \quad \mathrm{O}, \quad 0.3113 \% \mathrm{H})$ and concrete $(49.8 \% \mathrm{O}, 31.5 \% \mathrm{Si}, 0.6 \% \mathrm{H})$ respectively. A lower density of $7.4 \mathrm{~g} / \mathrm{cm}^{3}$ is used for steel shielding blocks where there are void spaces between layered steel plates. Figure 2 shows the high-energy neutron dose rate decrease with the increasing thickness of steel.

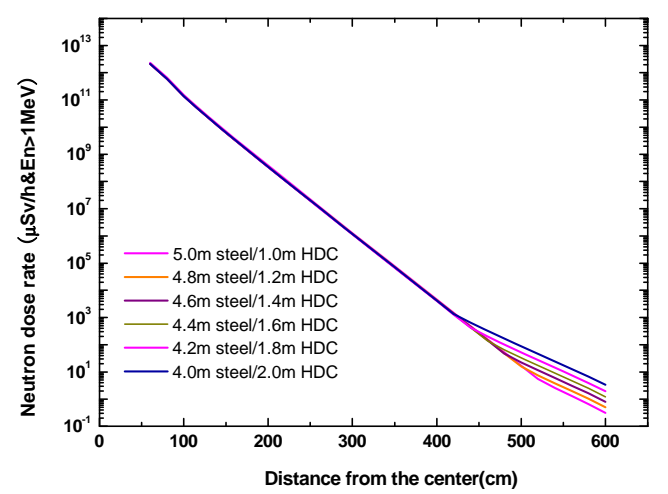

Figure 2. The high-energy neutron $(\mathrm{En}>1 \mathrm{MeV})$ dose rate decreases with the increasing thickness of steel. HDC in the figure means high density concrete, i.e. magnetite concrete.
For clarity, Table 1 gives the high-energy neutron $(E n>1 \mathrm{MeV})$ dose rate, the low-energy neutron (0.4 $\mathrm{eV} \sim 1 \mathrm{MeV}$ ) and the photon in the exterior shielding. We choose 4.8-meter-radius steel adding 1.2-meter-thick magnetite concrete as the shielding basis for the CSNS target station.

Table 1. The high-energy neutron $(\mathrm{En}>1 \mathrm{MeV})$, low-energy neutron $(1 \mathrm{MeV}>\mathrm{En}>0.4 \mathrm{eV})$ and total neutron dose rate in the exterior shielding. The unit is $\mu \mathrm{Sv} / \mathrm{h}$.

\begin{tabular}{|l|l|l|l|l|}
\hline Steel/HDC & $\begin{array}{l}\text { High-energy } \\
\text { neutron }\end{array}$ & $\begin{array}{l}\text { Low-energy } \\
\text { neutron }\end{array}$ & photon & Total \\
\hline $5.0 \mathrm{~m} / 1.0 \mathrm{~m}$ & 0.316 & 0.318 & 3.241 & 3.875 \\
\hline $4.8 \mathrm{~m} / 1.2 \mathrm{~m}$ & 0.505 & 0.522 & 0.811 & 1.838 \\
\hline $4.6 \mathrm{~m} / 1.4 \mathrm{~m}$ & 0.798 & 0.826 & 0.237 & 1.861 \\
\hline $4.4 \mathrm{~m} / 1.6 \mathrm{~m}$ & 1.23 & 1.27 & 0.0507 & 2.551 \\
\hline $4.2 \mathrm{~m} / 1.8 \mathrm{~m}$ & 1.94 & 2.00 & 0.0195 & 3.960 \\
\hline $4.0 \mathrm{~m} / 2.0 \mathrm{~m}$ & 3.32 & 3.44 & 0.0176 & 6.778 \\
\hline
\end{tabular}

However, ${ }^{56} \mathrm{Fe}$ ( iron is 91.7 percent ${ }^{56} \mathrm{Fe}$ ) has an important deficiency in shielding neutrons that its main property is to slow down neutron with energies above $847 \mathrm{keV}$ via inelastic scattering reactions, whilst below this threshold neutron can only lose its energy via elastic scattering [9]. Consequently, there is a build-up of neutrons below this energy. For this reason we consider a sandwich structure for the low-energy $(0.4 \mathrm{eV}<\mathrm{En}<1$ $\mathrm{MeV}$ ) neutron and photon shielding. Here we keep the total thickness of steel of this sandwich structure 4.8 meters, but we use the regular concrete instead of the high density concrete. For this structure we insert one 0.4 -meter-thick concrete layer in the steel shielding that is located distant from the centre 4 meter. We compare the dose rate between this sandwich structure and the integrated shielding that consist of 4.8-meter-radius steel adding 1.2-meter-thick regular concrete. The result shows that this sandwich structure is much more efficient for shielding photon than the integrated structure. The neutron dose rates in the exterior spheres are almost the same under these two conditions (see Figure 3 and 4). However, the low-energy neutron dose rate just below the sandwich structure decreases sharply. For further explanation, we give the neutron energy spectra in the areas from 1 to 10 under the two conditions. We find the intensity of thermal neutrons in the area 3 for the sandwich structure is higher than that for the integrated structure. However, if we focus on the area 6 , we find the intensity of neutrons for the sandwich structure is much lower than that for the integrated structure in the energy range from $10^{-9}$ to $10^{-1} \mathrm{MeV}$. This idea is very useful for reducing the residual radioactivity because major radioactivity in steel material is ${ }^{59} \mathrm{Fe}$ (half-life is about 44.5 days) that is mainly generated 
by the ${ }^{58} \mathrm{Fe}(\mathrm{n}, \mathrm{Y}){ }^{59} \mathrm{Fe}$ reactions with low-energy and



Figure 3. The dose rates of the sandwich structure. The areas 1 , 2, 7-10 are concrete shielding and the others are steel shielding.

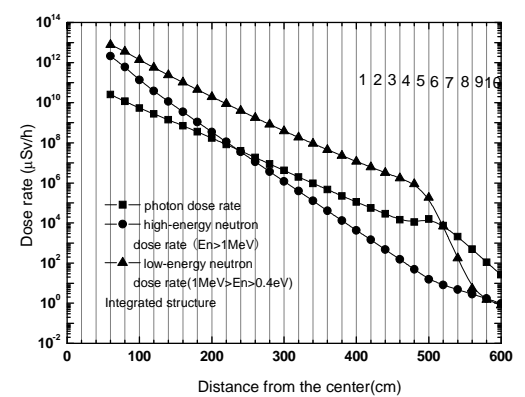

Figure 4 . The dose rates of the integrated structure. The areas 5-10 are concrete shielding and the others are steel shielding.

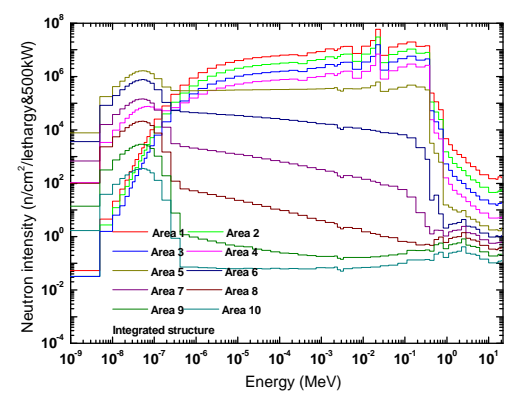

Figure 5. The neutron energy spectra of the area 1 to 10 for the integrated structure.



Figure 6 . The neutron energy spectra of the area 1 to 10 for the sandwich structure. thermal neutrons. If the area 6 is the maintenance area, the lower thermal neutron intensity means lower residual radioactivity. This idea may be adopted in the vertical direction after considering the appropriate positioning where the thin concrete layer is inserted.

\section{Three-dimension calculation models}

The structure of the CSNS target station is similar to those of SNS and J-PARC. The moderators are replaced vertically and the target is replaced horizontally. The three-dimension calculation model included a simplified target-moderator-reflector assembly, helium-vessel, neutron beam hole, shutters, shield blocks, and so on (see Figure 7). The CSNS target station uses tungsten as the target material, beryllium as the reflector material. In phase one CSNS has two hydrogen moderators and one water moderator. Twenty shutters begin from 2.25 meter to 4.25 meter relative to the TS center. Some importance gap and void spaces between these components are
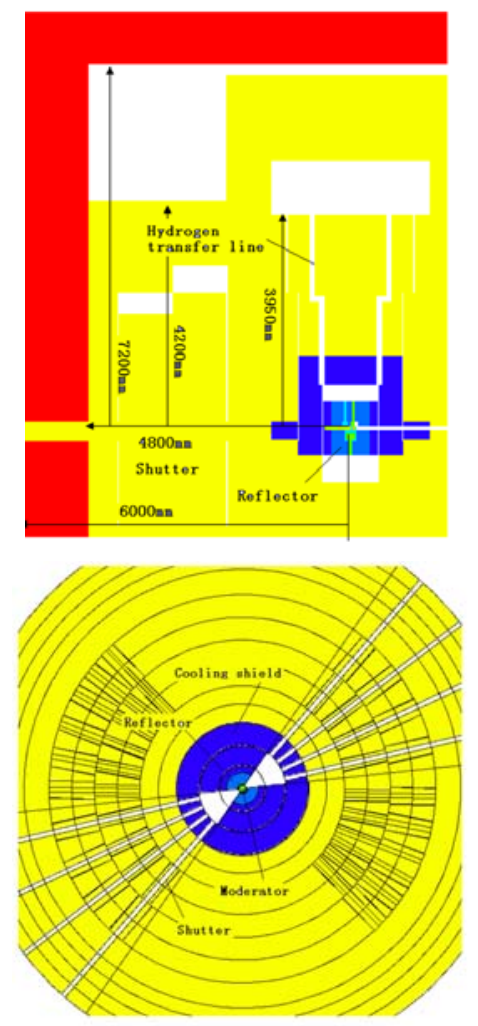

Figure 7. The vertical- and horizontal-cut view of 3-D MCNPX model (the shutter is open). The yellow shows steel and the red shows heavy density concrete. The densities of these materials are the same as mentioned above.

considered as following, (1) a cylindrical gap of $10 \mathrm{~mm}$ between out-reflector plug and helium vessel; (2) a cylindrical gap of $10 \mathrm{~mm}$ between helium vessel and shield blocks; (3) gaps of $10 \mathrm{~mm}$ between shield blocks and shutters. (4) a simplified cylindrical gap of $100 \mathrm{~mm}$ for the moderators transfer lines; (5) a void space of 400 
mm for shutter stroke and the step-type shutters are chosen to decrease the void space due to shutter traveling; (6) the shutters are open and the dimension of the beam hole of the shutter is $100 \mathrm{~mm} \times 100 \mathrm{~mm}$. We did not consider an importance gap between the outliner and the shielding because the gap is so large that we have to pour some granulated iron to avoid the stream effect.

The total dose map of neutrons and photons for this model is shown in Figure 8. It shows that the 4.8-meter-radius steel adding 1.2-meter-thick magnetite concrete can satisfy the shielding need. The dose rate in the CSNS user area is below $2.5 \mu \mathrm{Sv} / \mathrm{h}$. The area near the neutron beam hole will be shielded further in the neutron beam line shielding. Due to the streaming effect the dose rate on the top of the target station is about $3 \sim 8$ $\mu \mathrm{Sv} / \mathrm{h}$. This area is located in the target station high-bay and the dose limit for the high-bay is $25 \mu \mathrm{Sv} / \mathrm{h}$.

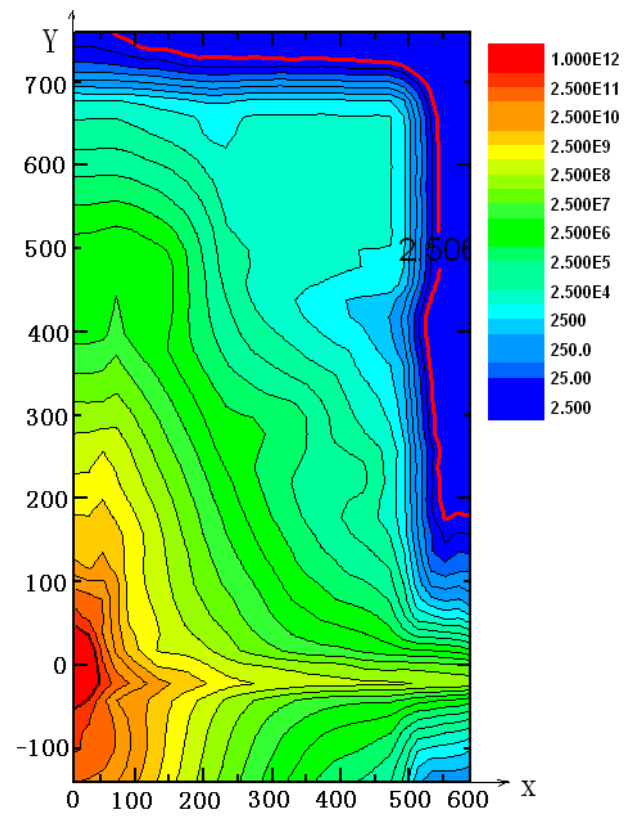

Figure 8. Calculated dose rate map (shutter is opened), the unit is $\mu \mathrm{Sv} / \mathrm{h}$, the dose rate of the red circle area on the top the target station is higher than $2.5 \mu \mathrm{Sv} / \mathrm{h}$. The units of the $\mathrm{X}$ and $\mathrm{Y}$ are centimeter.

In the present calculation model, we do not consider inserting concrete layers in the shielding. When other factors especially ventilation are checked on detail we will consider this sandwich structure.

\section{Conclusion and discussion}

We have calculated the shielding for CSNS TS with the Monte-Carlo code MCNPX2.5.0. Firstly one-dimension models with the different thickness of steel and magnetite concrete are calculated to determine the conceptual structure of CSNS TS under the condition that the energy of proton beam is $1.6 \mathrm{GeV}$ and the power is $500 \mathrm{~kW}$. We finally choose 4.8 -meter-radius steel structure of the CSNS TS. Then we calculate adding 1.2-meter-thick magnetite concrete as the basic three-dimension model of CSNS TS and some important gaps are considered in this model. The results show that 4.8-meter-radius steel adding 1.2-meter-thick magnetite concrete can satisfy the shielding need in the horizontal direction. The area near the neutron beam hole will be shielded further in the neutron beam line shielding. The dose rate on the top of CSNS TS is about $3 \sim 8 \mu \mathrm{Sv} / \mathrm{h}$ that is also below the dose rate limit $25 \mu \mathrm{Sv} / \mathrm{h}$. The sandwich structure is more efficient for photon shielding than the integrated structure.

\section{Acknowledgement}

We would like to thank Franz X.Gallmeier, Irina Popova and Wei Lu in SNS for very useful discussions for this design. This work was supported by the National Natural Science Foundation of China (Grant No.11174358, 11075203 and 91026009).

\section{References}

[1] Q.W.Yan, W.Yin and B.L.Yu, Optimized concept design of the target station of Chinese spallation neutron source, Journal of Nuclear Materials, 343 (2005), p.45.

[2] W.Yin, Q.Z.Yu, T.J.Liang and Q.W.Yan, Target station shielding, activation and decay heat analysis for CSNS, The 18th Meeting of the International Collaboration on Advanced Neutron Source (ICANS-XVIII), Dongguan, China, April 25-29, (2007).

[3] W.Yin, Q.Z.Yu, T.J.Liang and Y.X.Chen, 1st Workshop on Accelerator Radiation Induced Activation, PSI Proceedings 09-01 ISSN 1019-0643 (2008), p.176.

[4] DOORS3.2a One, Two- and Three Dimensional Discrete Ordinates Neutron/Photon Transport Code System, 2003.

[5] R.A.Lillie and F.X.Gallmeier, Hilo2k: A New Hilo library to $2 \mathrm{GeV}$, Oak Ridge National Laboratory, 2003.

[6] J.Wu, Y.X.Chen, W.J.Wang, W.Yin, T.J.Liang and X.J.Jia, The high-energy multi-group HEST1.0 library based on ENDF/B-VII.0 development, verification and preliminary application, Chinese Physics C, vol. 36(2012), pp.275.

[7] W.Yin, B.Zhang, T.J.Liang, Q.Z.Yu, Y.X.Chen and X.J.Jia, Shielding design for CSNS target station, The 19th Meeting of the International Collaboration on Advanced Neutron Source (ICANS-XIX), Grindelwald, Switzerland, March 8-12, (2010).

[8] MCNP/MCNPX CCC-730 Monte Carlo N-Particle Transport Code System Including MCNP5.1.40 and MCNPX2.5.0 and Data Libraries, 2005.

[9] Ralph H. Thomas (Chairman), Radiation protection for particle accelerator facilities, NCRP Report No.144 (2003), p.250. 